Check for updates

Cite this: RSC Adv., 2019, 9, 30905

Received 22nd August 2019

Accepted 24th September 2019

DOI: $10.1039 / c 9 r a 06628 d$

rsc.li/rsc-advances

\title{
Development of active and intelligent films based on cassava starch and Chinese bayberry (Myrica rubra Sieb. et Zucc.) anthocyanins $\uparrow$
}

\author{
Dawei Yun, $\stackrel{t}{+}^{a}$ Huahao Cai, $\stackrel{t}{t}^{a}$ Yunpeng Liu, ${ }^{a}$ Lixia Xiao, ${ }^{a}$ Jiangfeng Song*b \\ and Jun Liu (D)*a
}

\begin{abstract}
Chinese bayberry (Myrica rubra Sieb. et Zucc.) fruit is a functional food rich in anthocyanins. In this study, anthocyanin-rich bayberry extract (BBE) was added into cassava starch to develop food packaging films with antioxidant and $\mathrm{pH}$-sensitive properties. Results showed the main anthocyanin in BBE was cyanidin3-O-glucoside (95.6\%). The addition of $1 \mathrm{wt} \%$ of BBE into the film matrix resulted in a dense and compact internal microstructure, which greatly improved the water vapor permeability and tensile strength of the film. However, the addition of 2, 3 and $4 \mathrm{wt} \%$ of BBE into film matrix produced heterogeneous inner microstructures due to the formation of agglomerated BBE. The intermolecular interactions between BBE and the starch film matrix were through hydrogen binding. As compared with the starch film, starch-BBE films exhibited higher thicknesses, tensile strength, UV-vis light barrier and antioxidant properties. Moreover, starch-BBE films presented significant color changes when exposed to hydrogen chloride and ammonia gases. The $\mathrm{pH}$-sensitive starch-BBE films were able to monitor the freshness of pork. Our results suggested that starch-BBE films could be used as smart and active packaging materials in the food industry.
\end{abstract}

\section{Introduction}

Due to the awareness of environmental problems caused by plastic packaging materials, researchers have paid increasing attention to the development of biodegradable packaging films. ${ }^{1}$ Many naturally originating biopolymers including polysaccharides, proteins and lipids have been applied to prepare biodegradable packaging films. ${ }^{2}$ Among several biopolymers, starch is often selected to develop packaging films because it is abundant, cheap, edible and biodegradable. ${ }^{3}$ In general, the physicochemical and functional properties of starch-based films are closely related to its source (e.g. corn, wheat, rice, potato and cassava)., ${ }^{4,5}$ Cassava starch is regarded as a promising film-forming material because it has a low temperature of gelatinization and good gel stability. ${ }^{6}$ However, plain cassava starch films also have many drawbacks, such as lower mechanical, barrier and antioxidant properties. ${ }^{7}$ Therefore, many different natural ingredients including plant extracts, essential oils and other biopolymers have been incorporated

${ }^{a}$ College of Food Science and Engineering, Yangzhou University, Yangzhou 225127, PR China.E-mail: junliu@yzu.edu.cn

${ }^{b}$ Institute of Farm Product Processing, Jiangsu Academy of Agricultural Sciences, Nanjing 210014, PR China. E-mail: songjiangfeng102@163.com

$\dagger$ Electronic supplementary information (ESI) available. See DOI: 10.1039/c9ra06628d

$\ddagger$ These authors contributed equally to this work. into cassava starch films to improve the physical and functional properties of the films..$^{8-10}$

Anthocyanins are the most important pigments of vascular plants and are responsible for the pink, red, violet and blue colors of vegetables and fruits. ${ }^{11}$ Anthocyanins are glycosides and acylglycoside derivatives of six common anthocyanidins: cyanidin, delphinidin, malvidin, pelargonidin, peonidin and petunidin, which are classified according to the number and position of hydroxyl groups on the flavan nucleus. ${ }^{12}$ Notably, anthocyanins present different chemical forms and colors as the function of $\mathrm{pH} .{ }^{13}$ Thus, anthocyanin-rich films are considered as promising intelligent $\mathrm{pH}$ indicators to monitor the freshness of food since the process of food spoilage is usually accompanied by $\mathrm{pH}$ changes. ${ }^{14}$ Meanwhile, anthocyanins are one type of phenolic compounds with antioxidant and antibacterial activity, and are suitable to be incorporated into films to develop active packaging. ${ }^{15} \mathrm{Up}$ to now, several intelligent and active packaging films have been developed by incorporating anthocyanins from different sources (e.g. blueberry, grape and Lycium ruthenicum) into cassava starch. ${ }^{6,16-21}$ The physical and functional properties of anthocyanin-rich films are generally affected by the source and content of anthocyanins.

Bayberry (Myrica rubra Sieb. et Zucc.) belongs to the family of Myricaceae, which is widespread in tropical, subtropical and temperate areas of the world. ${ }^{22}$ China is the major commercial production area for bayberry. ${ }^{23}$ Chinese bayberries contain several kinds of nutritional components, such as carbohydrates, 
sugars, organic acids, proteins, minerals, vitamins and phenolic compounds. ${ }^{24}$ Among different components, phenolic compounds from Chinese bayberries including phenolic acids, anthocyanins and flavonols have been demonstrated to possess potent antioxidant activity. ${ }^{25-27}$ In addition, the major anthocyanin present in Chinese bayberries is identified as cyanidin-3-Oglucoside, which represents more than $95 \%$ of the total pigment content. ${ }^{28}$ Therefore, it is supposed that anthocyanins from Chinese bayberries are suitable to be used to develop active and intelligent packaging.

In this study, we aimed at developing active and intelligent packaging by adding anthocyanin-rich Chinese bayberry extract (BBE) into cassava starch for the first time. The effects of BBE content on the structural, physical, antioxidant and $\mathrm{pH}$ sensitive properties of starch-based films were determined. The developed films were also applied to monitor the freshness of pork.

\section{Materials and methods}

\subsection{Materials and chemical reagents}

Chinese bayberries ( $M$. rubra) were purchased from the local market (Yangzhou, China). AB-8 macroporous resin was purchased from Bohong Resin Technology Co., Ltd. (Tianjin, China). Cassava starch was obtained from Ganzhiyuan Sugar Industry Co., Ltd. (Nanjing, China). 2,2-Diphenyl-1picrylhydrazyl (DPPH) was purchased from Sigma Chemical Co. (MO, USA). Ethanol, glycerol, hydrochloric acid and ammonia were purchased from Sinopharm Chemical Reagent Co., Ltd. (Shanghai, China). All other reagents were of analytical grade.

\subsection{Extraction of anthocyanins}

Anthocyanins were extracted from Chinese bayberries according to the method of Qin et al. ${ }^{20}$ with some modifications. The fresh bayberries (250 g) were ground and extracted twice in $1000 \mathrm{~mL}$ of $80 \%$ ethanol solution containing $0.5 \% \mathrm{HCl}(\mathrm{v} / \mathrm{v})$ at $4{ }^{\circ} \mathrm{C}$ for $24 \mathrm{~h}$. All the extract solutions were combined and centrifuged at $8000 \mathrm{~g}$ for $20 \mathrm{~min}$ to remove debris. The supernatant was further purified on the column of AB-8 macroporous resin $(1.6 \mathrm{~cm} \times 60 \mathrm{~cm})$, which was continuously eluted with $80 \%$ ethanol solution containing $0.5 \% \mathrm{HCl}(\mathrm{v} / \mathrm{v})$. The collected eluate was concentrated by rotary evaporation at $40{ }^{\circ} \mathrm{C}$, followed by vacuum drying to obtain BBE powder.

\subsection{Characterization of anthocyanins}

The anthocyanin content in BBE was measured by $\mathrm{pH}$ differential assay. ${ }^{29}$ In addition, the composition of anthocyanins in the extract was further analyzed by Agilent 1200 HPLC system (Agilent Technologies, CA, USA) equipped with 6460 triple quadrupole mass spectrometer according to the chromatographic method of Zhang et al. ${ }^{30}$ The $\mathrm{pH}$-sensitivity of BBE was analyzed by dissolving $2 \mathrm{mg}$ BBE in $20 \mathrm{~mL}$ of different buffer solutions ( $\mathrm{pH} \mathrm{3-12)}$ for $5 \mathrm{~min}$, and the obtained BBE solutions were scanned on Lambda 35 ultraviolet-visible (UV- vis) spectrophotometer (PerkinElmer Inc., MA, USA) from 450 to $700 \mathrm{~nm}$.

\subsection{Development of the films}

Starch films with and without BBE were developed by the method of Stoll et al. ${ }^{31}$ Briefly, $4 \%$ of starch solution was prepared by dissolving $6.8 \mathrm{~g}$ of starch in $170 \mathrm{~mL}$ of deionized water at $90{ }^{\circ} \mathrm{C}$ with continuous stirring. Afterwards, the starch solution was mixed with different contents of $\operatorname{BBE}(0,1,2,3$ and $4 \mathrm{wt} \%$ ) and $25 \mathrm{wt} \%$ of glycerin based on the weight of starch. The resulting film-forming solutions were degassed, poured into Plexiglas plates $(24 \mathrm{~cm} \times 24 \mathrm{~cm})$ and then dried in a ventilated climate chamber at $30{ }^{\circ} \mathrm{C}$ and $50 \%$ relative humidity for $48 \mathrm{~h}$. The prepared films containing $0,1,2,3$ and $4 \mathrm{wt} \%$ of BBE were named as starch, starch-BBE I, starch-BBE II, starch-BBE III and starch-BBE IV films, respectively. All the films were stored at $20{ }^{\circ} \mathrm{C}$ and $50 \%$ relative humidity for at least $48 \mathrm{~h}$ before tests.

\subsection{Structural characterization of the films}

2.5.1. Scanning electron microscopy (SEM). The microstructure of the film was characterized by GeminiSEM 300 equipment (Carl Zeiss, Oberkochen, Germany). Film sample was fractured in liquid nitrogen, fixed on a stainless steel support with double-side conductive adhesive, sputtered with gold-palladium and analyzed on the SEM at $5 \mathrm{kV}$ with the magnification of $400 \times$.

2.5.2. Fourier transform infrared (FT-IR) spectroscopy. The FT-IR spectrum of the film was recorded on Varian 670 spectrometer (Varian Inc., CA, USA) in the wavenumber range of $4000-400 \mathrm{~cm}^{-1}$ with a total of 32 scans, operating at attenuated total reflection (ATR) mode.

2.5.3. X-ray diffraction (XRD). The crystalline character of the film was determined by D8 Advance X-ray diffractometer (Bruker AXS GmbH, Karlsruhe, Germany), conducting with Nifiltered $\mathrm{CuK} \alpha$ radiation at $40 \mathrm{~mA}$ and $40 \mathrm{kV}$. The XRD pattern was recorded from $2 \theta$ of $5-80^{\circ}$ with the scanning rate of $0.2^{\circ} \mathrm{s}^{-1}$.

\subsection{Determination of the physical properties of the films}

2.6.1. Color. The color parameters ( $L, a$ and $b$ ) of the film were determined by SC-80C colorimeter (Kangguang Instrument Co. Ltd, China). The total color difference $(\Delta E)$ and whiteness index (WI) of the film were calculated by the following formulas: ${ }^{18}$

$$
\begin{gathered}
\Delta E=\sqrt{\left(L^{*}-L\right)^{2}+\left(a^{*}-a\right)^{2}+\left(b^{*}-b\right)^{2}} \\
\mathrm{WI}=100-\sqrt{(100-L)^{2}+a^{2}+b^{2}}
\end{gathered}
$$

where $L^{*}, a^{*}$ and $b^{*}$ were the color parameters of standard white plate used for calibration, and $L, a$ and $b$ were the color parameters of the film.

2.6.2. Thickness. The thickness of the film was determined by Mitutoyo no. 293-766 digital micrometer (Tester Sangyo Co., Ltd., Tokyo, Japan) through randomly selecting ten different positions on each film. 
2.6.3. Water vapor permeability (WVP). The WVP of the film was determined by the method of Wang et al. ${ }^{32}$ Briefly, film sample $(6 \mathrm{~cm} \times 6 \mathrm{~cm})$ was sealed over $50 \mathrm{~mL}$ of centrifuge tube containing $30 \mathrm{~g}$ of dried silica gel. The centrifuge tube was then maintained at $25{ }^{\circ} \mathrm{C}$ in a desiccator containing distilled water. The centrifuge tube was weighed every $24 \mathrm{~h}$ for 7 days. The WVP of the film was calculated as follows:

$$
\mathrm{WVP}=\frac{W \times x}{t \times A \times \Delta P}
$$

where $W$ was the increased weight of the centrifuge tube $(\mathrm{g}), x$ was the film thickness (m), $t$ was the time (s) for the weight gain of the centrifuge tube, $A$ was the permeable area $\left(\mathrm{m}^{2}\right)$ of water vapor, and $\Delta P$ was the saturated vapor pressure at $25{ }^{\circ} \mathrm{C}$.

2.6.4. Light transmittance and opacity. The light transmittance of the film was measured by scanning film sample $(4 \mathrm{~cm} \times 0.9 \mathrm{~cm})$ on Lambda 35 UV-vis spectrophotometer (PerkinElmer Ltd., MA, USA) at the wavelength range from 200 to $800 \mathrm{~nm}$.

2.6.5. Mechanical properties. The mechanical properties of the film were determined by fixing the film strip $(6 \mathrm{~cm} \times 1 \mathrm{~cm})$ on TMS-Pro Texture Analyzer (Food Technology Co., VA, USA) at a crosshead speed of $6 \mathrm{~cm} \mathrm{~min}^{-1} .^{32}$ The tensile strength (TS) and elongation at break (EAB) of the film were calculated as follows:

$$
\begin{gathered}
\operatorname{TS}(\mathrm{MPa})=\frac{F}{x \times W} \\
\operatorname{EAB}(\%)=\frac{\Delta L}{L_{0}} \times 100
\end{gathered}
$$

where $F$ was the stress for film fracture (N), $x$ was film thickness (mm), $W$ was film width ( $\mathrm{mm}), \Delta L$ was the increased length at film break (mm) and $L_{0}$ was the initial film length (mm).

2.6.6. Thermogravimetric analysis (TGA). The thermal stability of the film was determined by heating film sample (2 $\mathrm{mg}$ ) from 30 to $800^{\circ} \mathrm{C}$ on Pyris 1 instrument (PerkinElmer Inc., MA, USA) under nitrogen atmosphere.

\subsection{Determination of the antioxidant property of the films}

The antioxidant property of the film was determined by DPPH radical scavenging test. ${ }^{33}$ Briefly, different amounts $(4,8,12,16$ and $20 \mathrm{mg}$ ) of the film sample were weighed and individually placed in the test tubes. Then, $4 \mathrm{~mL}$ of $100 \mu \mathrm{M}$ DPPH methanol solution was added into each tube and reacted with film sample at $25{ }^{\circ} \mathrm{C}$ for $1 \mathrm{~h}$ in the dark. The absorbance of the reaction solution was measured at $517 \mathrm{~nm}$. The DPPH radical scavenging activity of the film was calculated as follows:

$$
\text { DPPH radical scavenging activity }(\%)=\frac{A_{0}-A_{1}}{A_{0}} \times 100
$$

where $A_{0}$ was absorbance of blank (without film) and $A_{1}$ was absorbance of reaction solution containing film.

\subsection{Determination of $\mathbf{p H}$-sensitive property of the films}

The $\mathrm{pH}$ sensitivity property of the film was determined by the method of $\mathrm{Wu}$ et $a l^{34}$ with some modifications. Film samples
$(1.5 \mathrm{~cm} \times 1.5 \mathrm{~cm})$ were divided into two groups and placed in the headspace of the sealed containers loaded with $0.4 \mathrm{M}$ hydrogen chloride and $0.4 \mathrm{M}$ ammonia water, respectively. The color changes of the film sample were recorded every 5 min for $60 \mathrm{~min}$. The color parameters of the film sample at different times were determined as described in Section 2.6.1.

\subsection{Application of the films for monitoring the freshness of pork}

The colorimetric films were applied to monitor the freshness of pork. ${ }^{20}$ Briefly, $30 \mathrm{~g}$ fresh pork was placed in a Petri dish and covered with polyethylene film. Film sample $(2 \mathrm{~cm} \times 2 \mathrm{~cm})$ was fixed to the headspace of Petri dish. Then, the Petri dish was stored at $25{ }^{\circ} \mathrm{C}$ for $48 \mathrm{~h}$. The color changes of film sample were recorded periodically. Meanwhile, the $\mathrm{pH}$ and total volatile basic nitrogen (TVB-N) values of the pork were determined based on the Chinese standards of GB 5009.237-2016 and GB 5009.228-2016, respectively.

\subsection{Statistical analysis}

Duncan test and one-way analysis of variance were carried out by SPSS 13.0 software package (SPSS Inc., IL, USA). Difference was considered as statistically significant if $p<0.05$.

\section{Results and discussion}

\subsection{Characterization of anthocyanins in BBE}

Anthocyanins were extracted from Chinese bayberries and the total anthocyanin content in the extract was determined as $273 \mathrm{mg} \mathrm{g}^{-1}$ by $\mathrm{pH}$-differential assay. The obtained extract was further separated by HPLC and identified by their mass spectra. As shown in Table 1, three kinds of anthocyanins including delphinidin-3-O-glucose (3.7\%), cyanidin-3-O-glucoside (95.6\%) and pelargonidin-3-O-glucose $(0.7 \%)$ were detected, indicating cyanidin-3-O-glucoside was the main anthocyanin in BBE. This was consistent with the results of other researchers, ${ }^{22,35}$ who found cyanidin-3-O-glucoside was the primary anthocyanin in Chinese bayberry. Zhang et al. ${ }^{36}$ suggested the composition of anthocyanins was closely related with the cultivar of Chinese bayberries.

\subsection{Color variations of BBE in different buffer solutions}

The color variations of BBE in different buffer solutions were shown in Fig. 1A. Significant color changes were observed in BBE solutions as the function of $\mathrm{pH}$. When $\mathrm{pH}$ value was lower than 7, the color of BBE solution was red/pink. At the neutral condition ( $\mathrm{pH} 7$ ), a brown color was observed for BBE solution. However, the color of BBE solution turned purple at $\mathrm{pH}$ 8-10 and yellow at $\mathrm{pH} 11-12$. Corresponding to the color variations of BBE in different buffer solutions, the maximum absorption peak of BBE solution gradually moved from $510 \mathrm{~nm}$ towards $590 \mathrm{~nm}$ with the increase of $\mathrm{pH}$ value (Fig. 1B). The color variations and corresponding bathochromic shift in the maximum absorption peak of BBE solution were caused by the structure transformation of anthocyanins at different $\mathrm{pH}$ conditions. ${ }^{37}$ Anthocyanins present the form of red flavylium cation in strong 
Table 1 The composition of anthocyanins in BBE as analyzed by HPLC-MS

\begin{tabular}{lllrr}
\hline Peak & $\begin{array}{l}\text { Retention time } \\
(\mathrm{min})\end{array}$ & Anthocyanins & {$[\mathrm{M}]^{+}(\mathrm{m} / \mathrm{z})$} & $\begin{array}{l}\text { Relative content } \\
(\%)\end{array}$ \\
\hline 1 & 1.85 & Delphinidin-3-O-glucose & 465 & 3.7 \\
2 & 5.92 & Cyanidin-3-O-glucoside & 449 & 95.6 \\
3 & 7.56 & Pelargonidin-3-O-glucose & 433 & 0.7
\end{tabular}

acidic conditions, which gradually transform into pink carbinol pseudobase, purple quinonoidal anhydrobase and finally light yellow chalcone in strong alkaline conditions. ${ }^{38}$ Similarly, other researchers also found anthocyanins from different sources (e.g. L. ruthenicum and purple sweet potato) exhibited color variations in different buffer solutions..$^{14,20}$

\subsection{Microstructure of starch-BBE films}

The cross-sectional morphology of starch film and starch-BBE films is shown in Fig. 2. The cross-section of starch film was relatively smooth without significant wrinkles and protrusions, indicating that glycerol and starch were uniformly distributed in the film matrix. As compared to starch film, starch-BBE I film showed a more flat and dense cross-section. This suggested a low content (1 wt\%) of BBE could be well mixed with other film components (e.g. starch and glycerol). The dense internal microstructure of starchBBE I film was benefit to improve the water vapor barrier and mechanical properties of the film. However, starch-BBE II, III and IV films exhibited much rougher cross-sections as compared to starch and starch-BBE I films. The rougher cross-sections of starch films containing 2, 3 and $4 \mathrm{wt} \%$ of BBE were probably caused by the agglomerated BBE particles that occurred when the content of BBE exceeded $1 \mathrm{wt} \%$. The heterogeneous inner microstructures of these films indicated the compactness of film network was destroyed by excessive extract. Similarly, Qin et $a .^{20}$ found the cross-sectional morphology of starch-based films was closely related to the content of L. ruthenicum anthocyanins. The films presented dense inner microstructures when 1 and $2 \mathrm{wt} \%$ of anthocyanins was added, whereas the films exhibited a rough cross-section when $4 \mathrm{wt} \%$ of anthocyanins were incorporated. Zhang et al. ${ }^{39}$ also

(A)
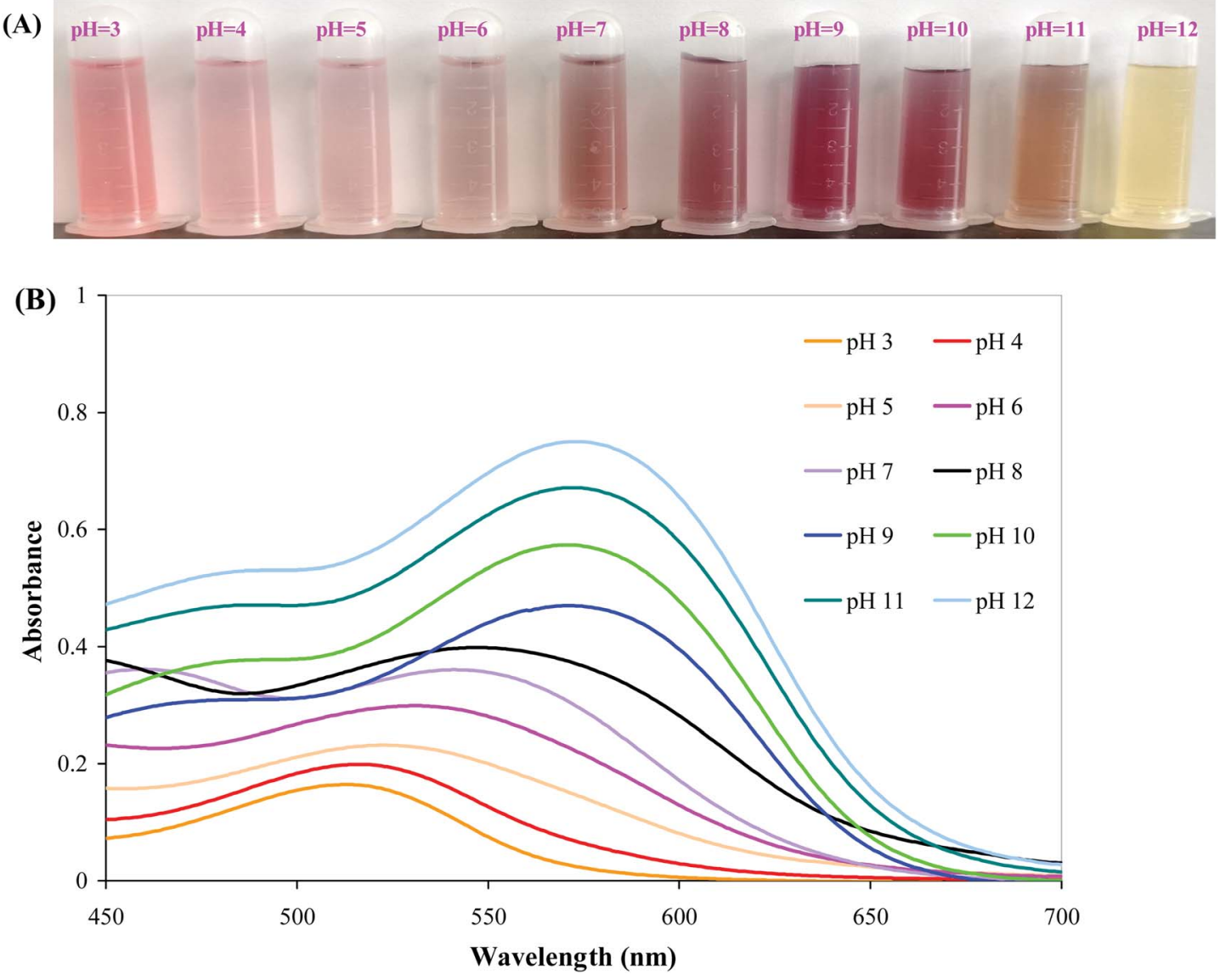

Fig. 1 Color variations (A) and UV-vis spectra (B) of BBE in different buffer solutions (pH 3 to 12). 

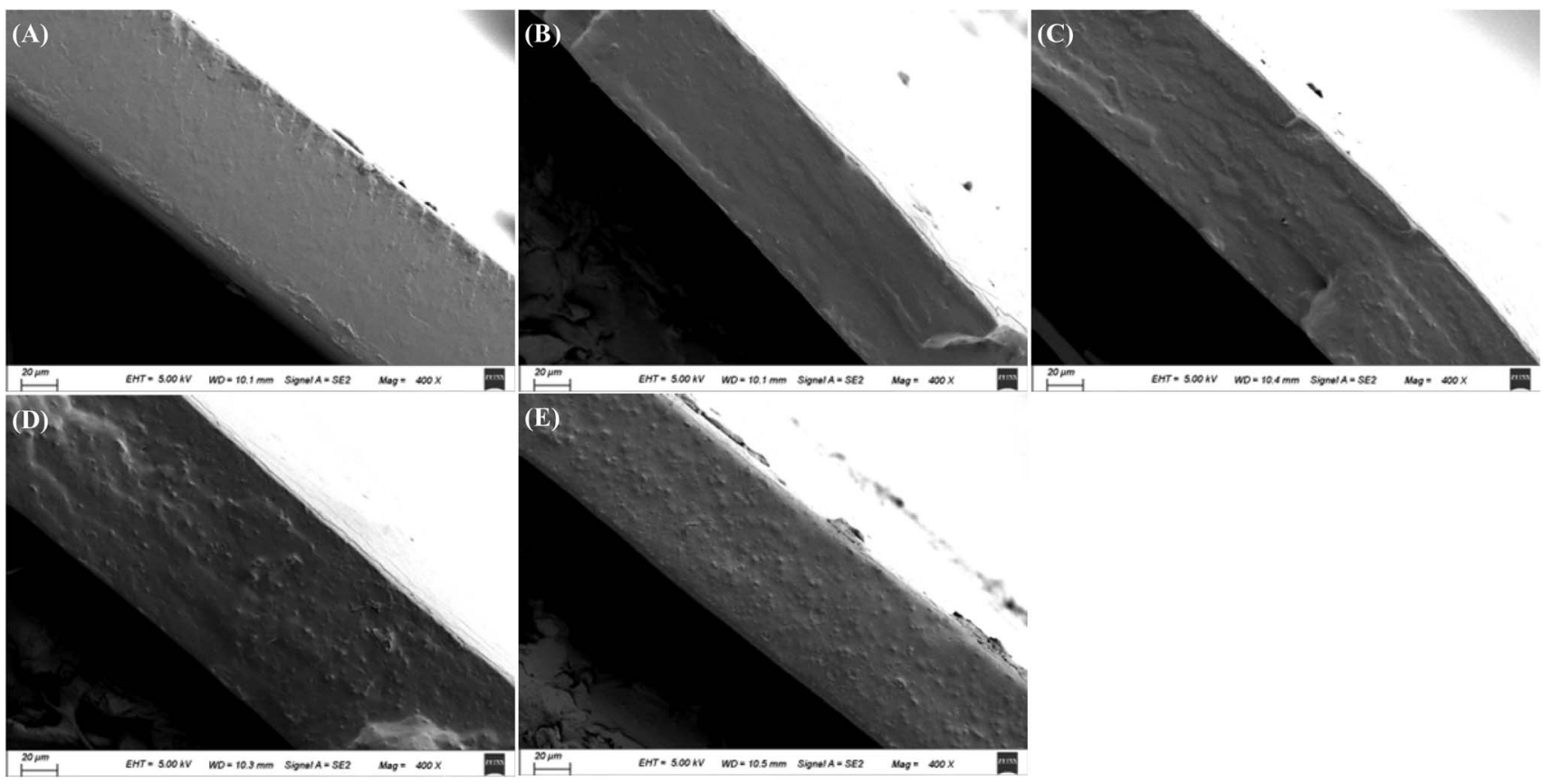

Fig. 2 The cross-section morphology of starch (A), starch-BBE I (B), starch-BBE II (C), starch-BBE III (D) and starch-BBE IV (E) films.

reported that starch-chitosan blend film containing $2.5 \mathrm{wt} \%$ of roselle anthocyanins had heterogeneous internal microstructures, revealing anthocyanins could destroy the ordered arrangement between starch and chitosan.

\subsection{FT-IR spectra of starch-BBE films}

The intermolecular interactions between starch and BBE were revealed by ATR FT-IR technique and shown in Fig. 3. BBE presented characteristic bands of polyphenols at $3258 \mathrm{~cm}^{-1}\left(\mathrm{O}^{-}\right.$ $\mathrm{H}$ stretching), $1604 \mathrm{~cm}^{-1}$ ( $\mathrm{C}=\mathrm{C}$ stretching of aromatic ring) and $1015 \mathrm{~cm}^{-1}$ (C-H deformation of aromatic ring). ${ }^{40}$ Starch film exhibited bands at $3317 \mathrm{~cm}^{-1}(\mathrm{O}-\mathrm{H}$ stretching $), 2926 \mathrm{~cm}^{-1}(\mathrm{C}-\mathrm{H}$ stretching of glucose residues), $1645 \mathrm{~cm}^{-1}$ (O-H bending of bound water), 994 to $1150 \mathrm{~cm}^{-1}$ (pyranose ring of glucose residues). ${ }^{41}$ The FT-IR spectra of starch-BBE films were somewhat different from that of starch film. On one hand, the $\mathrm{O}-\mathrm{H}$ stretching of starch at $3317 \mathrm{~cm}^{-1}$ decreased and shifted to $3304 \mathrm{~cm}^{-1}$ after the incorporation of BBE, which was caused by the formation of hydrogen binding between BBE and starch that reduced the number of available hydroxyl groups. Similar results were also observed when anthocyanin-rich Jamaica flower extract was incorporated into starch based films. ${ }^{42}$ On the other hand, the $\mathrm{O}-\mathrm{H}$ bending of bound water at $1645 \mathrm{~cm}^{-1}$ of starch slightly shifted to $1643 / 1642 \mathrm{~cm}^{-1}$ in starch-BBE films, suggesting the interactions (hydrogen binding) occurred between BBE and bound water. Choi et al. ${ }^{14}$ also observed that the band of potato starch/agar blend film at $1645 \mathrm{~cm}^{-1}$ shifted to a lower wavenumber $\left(1617 \mathrm{~cm}^{-1}\right)$ after the addition of anthocyanin-rich purple sweet potato extract, which was attributed to hydrogen binding between film matrix and the extract. Above results suggested that BBE was immobilized in the film matrix.

\subsection{XRD patterns of starch-BBE films}

The intermolecular interactions between BBE and starch can be also studied by XRD. As shown in Fig. 4, the XRD profile of BBE displayed a big broad peak at $2 \theta=24^{\circ}$. This suggested BBE was in the amorphous state. Similar results were observed in other anthocyanin-rich plant extracts..$^{20,40}$ As for starch film, it showed a broad peak at around $2 \theta=20^{\circ}$. The formation of amorphous state in starch film was because the original semi-crystalline regions of starch granules were destroyed by heating during gelatinization. ${ }^{43}$ When BBE was added, the amorphous peak of starch film was almost unchanged. This indicated BBE was well dispersed in the film matrix. Notably, a new crystalline peak appeared at about $2 \theta=24^{\circ}$ when 2,3 and $4 \mathrm{wt} \%$ of BBE were incorporated into starch film, which well corresponded to the amorphous peak position of BBE. The increased crystalline state in starch-BBE films was because of the formation of BBE agglomerates that exhibited the crystalline character. However, Zhai et al. ${ }^{43}$ reported that the incorporation of roselle anthocyanins decreased the crystalline state of starch/polyvinyl alcohol films, which could be explained by the hydrogen bonding formed between anthocyanins and film matrix. Qin et al. ${ }^{20}$ found the amorphous peak intensity of starch film increased after the addition of L. ruthenicum anthocyanins, which was probably because the hydrogen bonding formed between starch and anthocyanins resulted in three dimensional network. Above results suggested the crystallinity of anthocyanin-rich starchbased films were affected by the nature of anthocyanins.

\subsection{Colors of starch-BBE films}

Color is the most intuitive property of the film, which greatly affects the acceptance degree of consumers. ${ }^{44}$ As shown in 


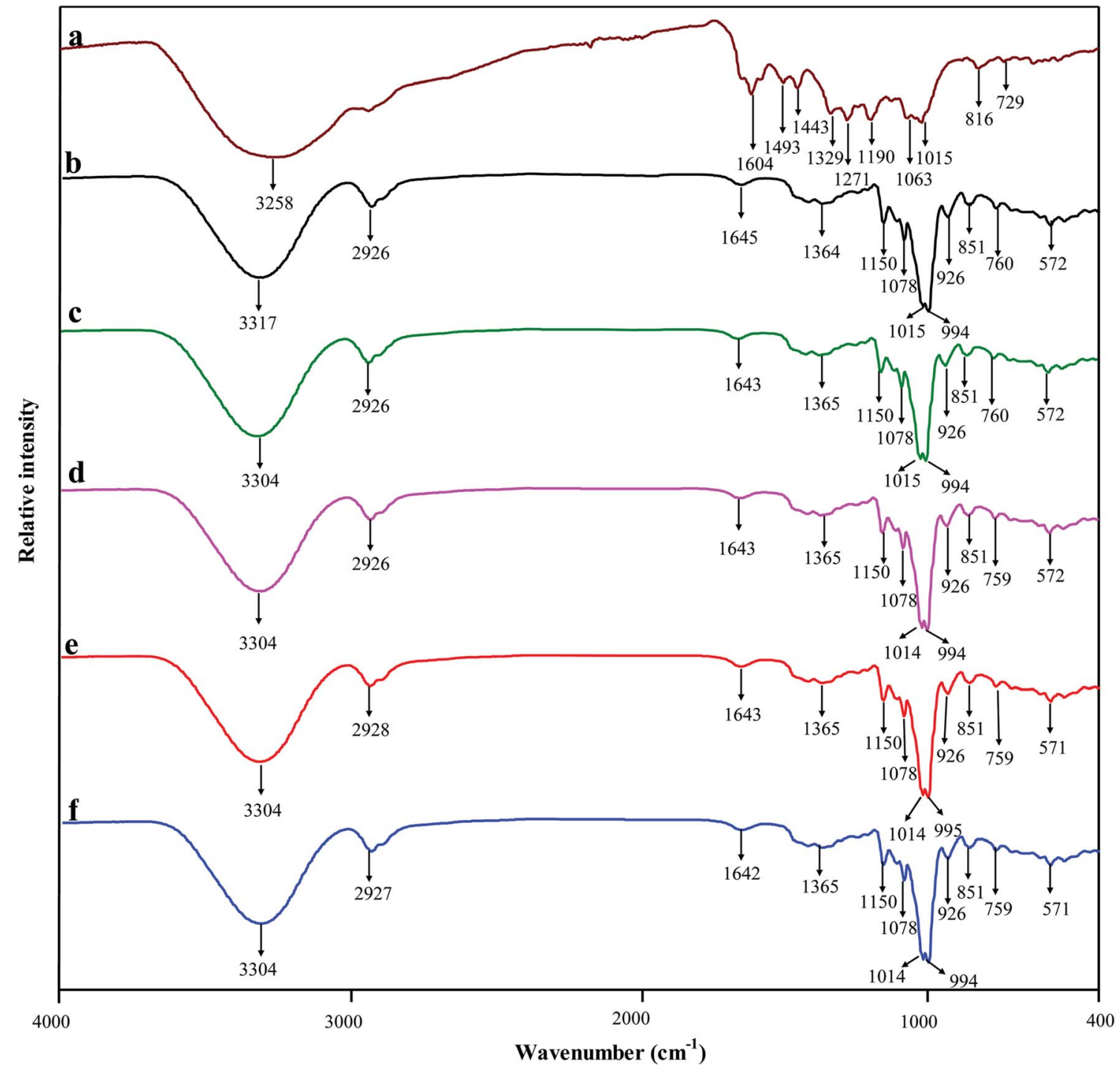

Fig. 3 FT-IR spectra of BBE powder (a), and starch (b), starch-BBE I (c), starch-BBE II (d), starch-BBE III (e) and starch-BBE IV (f) films.

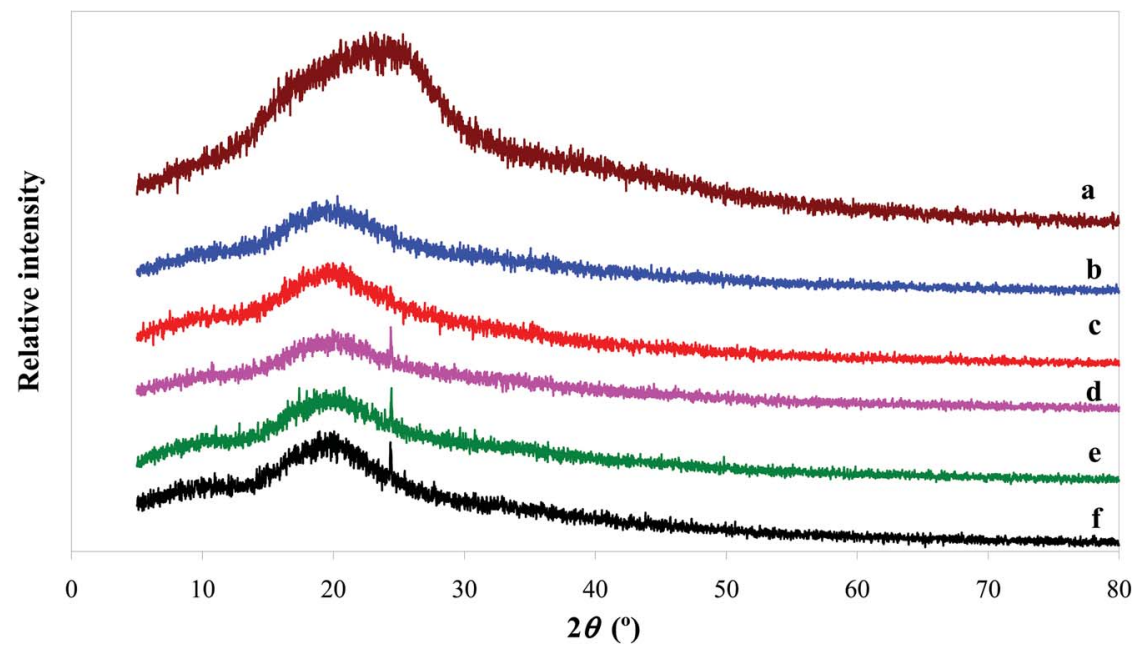

Fig. 4 XRD patterns of BBE powder (a), and starch (b), starch-BBE I (c), starch-BBE II (d), starch-BBE III (e) and starch-BBE IV (f) films. 
(A)

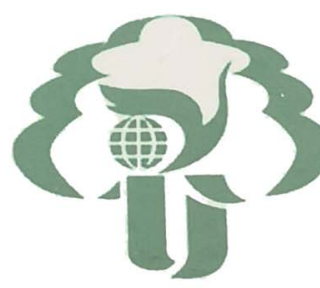

Starch film

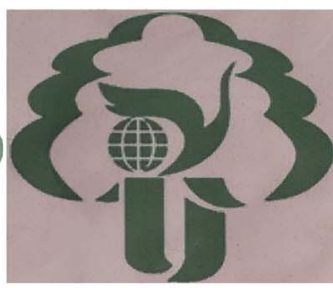

Starch-BBE I film

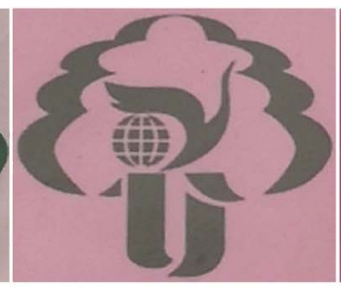

Starch-BBE II film

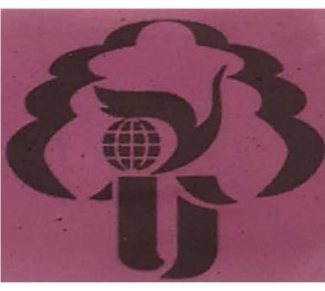

Starch-BBE III film

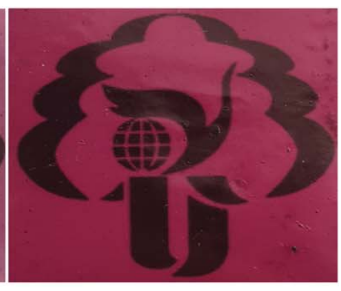

Starch-BBE IV film

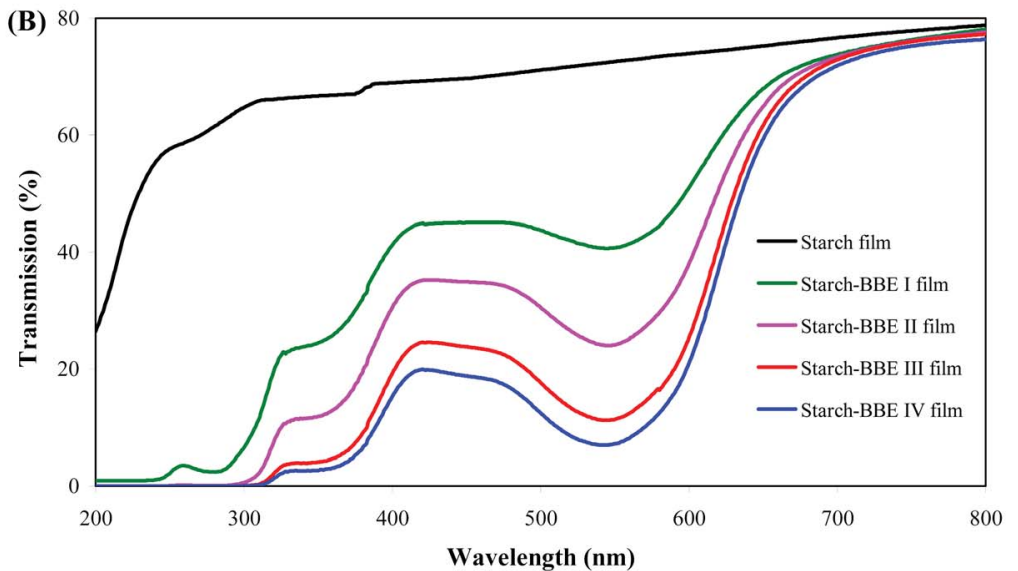

Fig. 5 Physical appearances (A) and UV-vis light transmittance (B) of starch and starch-BBE films.

Fig. 5A, the colors of starch-BBE films were significantly different from that of starch film. Starch film was colorless whereas starch-BBE films were purplish red. In addition, the colors of starch-BBE films deepened with the increase of BBE content. The purplish red colors of starch-BBE films were attributed to the presence of anthocyanins in BBE. The similar colors were observed in starch films containing L. ruthenicum anthocyanins. ${ }^{20}$ Table 2 summarizes the color parameters of different films. Starch-BBE films showed higher $a$ values than starch film, which was consistent with the red color of the films. The $a$ values of starch-BBE films significantly increased when BBE content increased, which agreed with the gradually deepened color of starch-BBE films. In addition, the $\Delta E$ values of starch-BBE films significantly increased with the increase of $\mathrm{BBE}$ content, indicating starch-BBE films became more colored. However, the $L$ and WI values of starch-BBE films decreased as BBE content increased, suggesting the tendency of the films toward dark. Above results indicated the color parameters of starch-BBE films were greatly affected by the content of the extract. Similar changes in the color parameters were obtained when anthocyanin-rich blackberry pulp and red rice flour were added into starch films. ${ }^{\mathbf{4 5 4}}$

\subsection{Thicknesses of starch-BBE films}

Film thickness is an important parameter that directly affects the mechanical strength, WVP and light transmittance of the film. ${ }^{47}$ As presented in Table 3, the thickness of starch and starch-BBE films varied from 0.076 to $0.089 \mathrm{~mm}$. A significant enhancement in film thickness was observed when BBE content exceeded $1 \mathrm{wt} \%$, which was because the high contents of the extract created heterogeneous film network that made the films became thicker. However, the incorporation of $1 \mathrm{wt} \%$ of $\mathrm{BBE}$ did not significantly change the thickness of the film. This result was in agreement the SEM observation that low content of BBE was well compatible with film matrix, which produced a dense internal microstructure. Qin et $a .^{20}$ also found the thickness of anthocyanin-rich starch films was affected by the content of anthocyanins.

Table 2 Color values including $L, a, b, \Delta E$ and WI of starch and starch-BBE films ${ }^{a}$

\begin{tabular}{|c|c|c|c|c|c|}
\hline Films & $L$ & $a$ & $b$ & $\Delta E$ & WI \\
\hline Starch film & $94.35 \pm 0.05^{\mathrm{a}}$ & $-0.53 \pm 0.01^{\mathrm{d}}$ & $-2.16 \pm 0.06^{\mathrm{a}}$ & $1.53 \pm 0.04^{\mathrm{e}}$ & $93.92 \pm 0.07^{\mathrm{a}}$ \\
\hline Starch-BBE I film & $70.09 \pm 0.83^{\mathrm{b}}$ & $14.69 \pm 0.66^{\mathrm{c}}$ & $-7.43 \pm 0.21^{\mathrm{c}}$ & $28.09 \pm 1.08^{\mathrm{d}}$ & $65.86 \pm 1.06^{b}$ \\
\hline Starch-BBE II film & $50.31 \pm 0.71^{\mathrm{c}}$ & $30.00 \pm 0.47^{\mathrm{b}}$ & $-10.31 \pm 0.10^{\mathrm{d}}$ & $53.20 \pm 0.85^{\mathrm{c}}$ & $41.05 \pm 0.85^{\mathrm{c}}$ \\
\hline Starch-BBE III film & $40.72 \pm 1.56^{\mathrm{d}}$ & $33.40 \pm 0.23^{\mathrm{a}}$ & $-6.87 \pm 1.13^{\mathrm{c}}$ & $62.57 \pm 1.09^{b}$ & $31.60 \pm 1.13^{d}$ \\
\hline Starch-BBE IV film & $36.84 \pm 0.16^{\mathrm{e}}$ & $32.96 \pm 0.18^{\mathrm{a}}$ & $-4.10 \pm 0.25^{\mathrm{b}}$ & $66.59 \pm 0.22^{\mathrm{a}}$ & $28.64 \pm 0.21^{\mathrm{e}}$ \\
\hline
\end{tabular}

${ }^{a}$ Values are given as mean $\pm \mathrm{SD}(n=3)$. Different letters in the same column indicate significantly different $(p<0.05)$. 
Table 3 Thicknesses, WVP, TS and EAB of starch and starch-BBE films ${ }^{a}$

\begin{tabular}{|c|c|c|c|c|}
\hline Films & Thickness (mm) & WVP $\left(\times 10^{-10} \mathrm{~g} \mathrm{~m}^{-1} \mathrm{~s}^{-1} \mathrm{~Pa}\right)$ & TS (MPa) & $\mathrm{EAB}(\%)$ \\
\hline Starch film & $0.076 \pm 0.001^{\mathrm{b}}$ & $1.084 \pm 0.021^{\mathrm{c}}$ & $8.41 \pm 0.85^{\mathrm{d}}$ & $4.26 \pm 0.59^{\mathrm{a}}$ \\
\hline Starch-BBE I film & $0.078 \pm 0.002^{\mathrm{b}}$ & $0.984 \pm 0.068^{\mathrm{d}}$ & $11.74 \pm 1.47^{\mathrm{a}}$ & $4.19 \pm 0.09^{\mathrm{a}, \mathrm{b}}$ \\
\hline Starch-BBE III film & $0.087 \pm 0.006^{\mathrm{a}}$ & $1.164 \pm 0.033^{\mathrm{b}}$ & $10.47 \pm 0.31^{\mathrm{b}}$ & $3.83 \pm 0.27^{\mathrm{b}}$ \\
\hline Starch-BBE IV film & $0.088 \pm 0.001^{\mathrm{a}}$ & $1.243 \pm 0.051^{\mathrm{a}}$ & $9.39 \pm 0.20^{\mathrm{c}}$ & $3.72 \pm 0.14^{\mathrm{b}}$ \\
\hline
\end{tabular}

${ }^{a}$ Values are given as mean $\pm \mathrm{SD}(n=10$ for thickness, $n=3$ for WVP, and $n=6$ for TS and EAB). Different letters in the same column indicate significantly different $(p<0.05)$.

\subsection{WVP of starch-BBE films}

WVP is a vital barrier parameter for food packaging films to prevent moisture transfer between food and the environment. As shown in Table 3, the WVP value of starch-BBE I film (0.984 $\times 10^{-10} \mathrm{~g} \mathrm{~m}^{-1} \mathrm{~s}^{-1} \mathrm{~Pa}$ ) was lower than that of starch film (1.084 $\times 10^{-10} \mathrm{~g} \mathrm{~m}^{-1} \mathrm{~s}^{-1} \mathrm{~Pa}$ ), which was because the incorporation of a low content of BBE had created more dense and compact network to inhibit moisture transfer. In addition, the intermolecular interactions (i.e. hydrogen binding) between starch and BBE could reduced the availability of hydrophilic hydroxyl groups in starch-BBE films, thereby reducing the affinity of the films toward water vapor. ${ }^{20}$ However, the WVP values of starchBBE films significantly increased when the content of BBE exceeded $2 \mathrm{wt} \%$, which was because the excessive extract formed aggregates and produced more free volumes in the film network to facilitate moisture transfer. Luchese et al. ${ }^{6}$ found the incorporation of anthocyanin-rich blueberry residue significantly increased the WVP of starch film, which was due to the blueberry residue addition destructed the compactness of the film. Similarly, Nogueira et al. ${ }^{45}$ reported the addition 20, 30 and $40 \mathrm{wt} \%$ of blackberry pulp into starch films remarkably enhanced the WVP of the films, which was because blackberry pulp promoted the discontinuity of polymer matrix. Our results suggested the water vapor barrier property of starch film could be improved by incorporating $1 \mathrm{wt} \%$ of BBE.

\subsection{Light transmittance of starch-BBE films}

UV-vis light barrier property of the film is very important for light-sensitive food packaging. UV-vis light can accelerate food degradation and oxidation, leading to nutrient destruction, color loss and toxic substance formation. ${ }^{48}$ As shown in Fig. 5B, starch-BBE films showed significantly lower light transmittance in both UV and visible wavelength ranges as compared to starch film, suggesting starch-BBE films possessed higher UV-vis light barrier ability than starch film. The UV-vis light barrier ability of starch-BBE films was mainly attributed to the aromatic rings in the anthocyanins that could absorb UV-vis light. ${ }^{49}$ In addition, the UV-vis light barrier ability of starch-BBE films was positively correlated with BBE content in the films. Luchese et al. ${ }^{19}$ and Gutiérrez et al. ${ }^{50}$ also found the UV-vis light barrier property of anthocyanin-rich starch films was elevated with the increase of the content of anthocyanins. Notably, a significant reduction in the light transmittance at about $550 \mathrm{~nm}$ was observed for
starch-BBE films, which corresponded to the visible absorption peak of anthocyanins. Similar phenomena were reported in starch films containing L. ruthenicum anthocyanins. ${ }^{20}$

\subsection{Mechanical property of starch-BBE films}

The mechanical properties including TS and EAB of different films are shown in Table 3. Starch-BBE films had relatively higher TS values (9.39-11.74 MPa) than starch film (8.41 MPa). The increased TS in starch-BBE films was because the abundant hydroxyl groups in anthocyanins could form hydrogen bonding with the hydroxyl groups in starch, resulting in stronger interfacial adhesion between starch and BBE. ${ }^{51}$ However, the TS of starch-BBE films gradually decreased as the BBE content increased, which was due to the excessive extract formed aggregates that interrupted the compactness of film network. ${ }^{43}$ The EAB values of starch-BBE I and II films (4.01-4.19\%) were not significantly different from that of starch film (4.26\%). Only starch-BBE III and IV films exhibited lower EAB values (3.72$3.83 \%$ ) than starch film. This was probably because the BBE aggregates limited the motion of polymer chains, resulting in the decrease of film flexibility. The similar changes in the TS and $\mathrm{EAB}$ values were found when $L$. ruthenicum anthocyanins were added into starch films. ${ }^{20}$ Zhai et $a .^{43}$ also reported the mechanical properties of starch/polyvinyl alcohol films were affected by the content of roselle anthocyanins. However, they found the TS values of the films decreased whereas the EAB values of the films increased with the increase of anthocyanin content. Therefore, the mechanical properties of anthocyaninrich starch films could be related with the nature of anthocyanins.

\subsection{Thermal property of starch-BBE films}

The TGA and DTG profiles showed all the films were degraded in three stages (Fig. 6). For starch film, the first weight loss stage $\left(30-185^{\circ} \mathrm{C}\right)$ was associated with the release of free water in the film. The second weight loss stage $\left(185-260{ }^{\circ} \mathrm{C}\right)$ was related to the evaporation of glycerol. The third weight loss stage (260-800 ${ }^{\circ} \mathrm{C}$ ) was ascribed to the depolymerization and decomposition of starch molecules. ${ }^{52}$ However, the TGA and DTG profiles of starch-BBE films almost overlapped with those of starch film, indicating the incorporation of BBE did not significantly alter the thermal property of the films. Similarly, Qin et al. ${ }^{20}$ also reported the thermal property of starch-based films was 

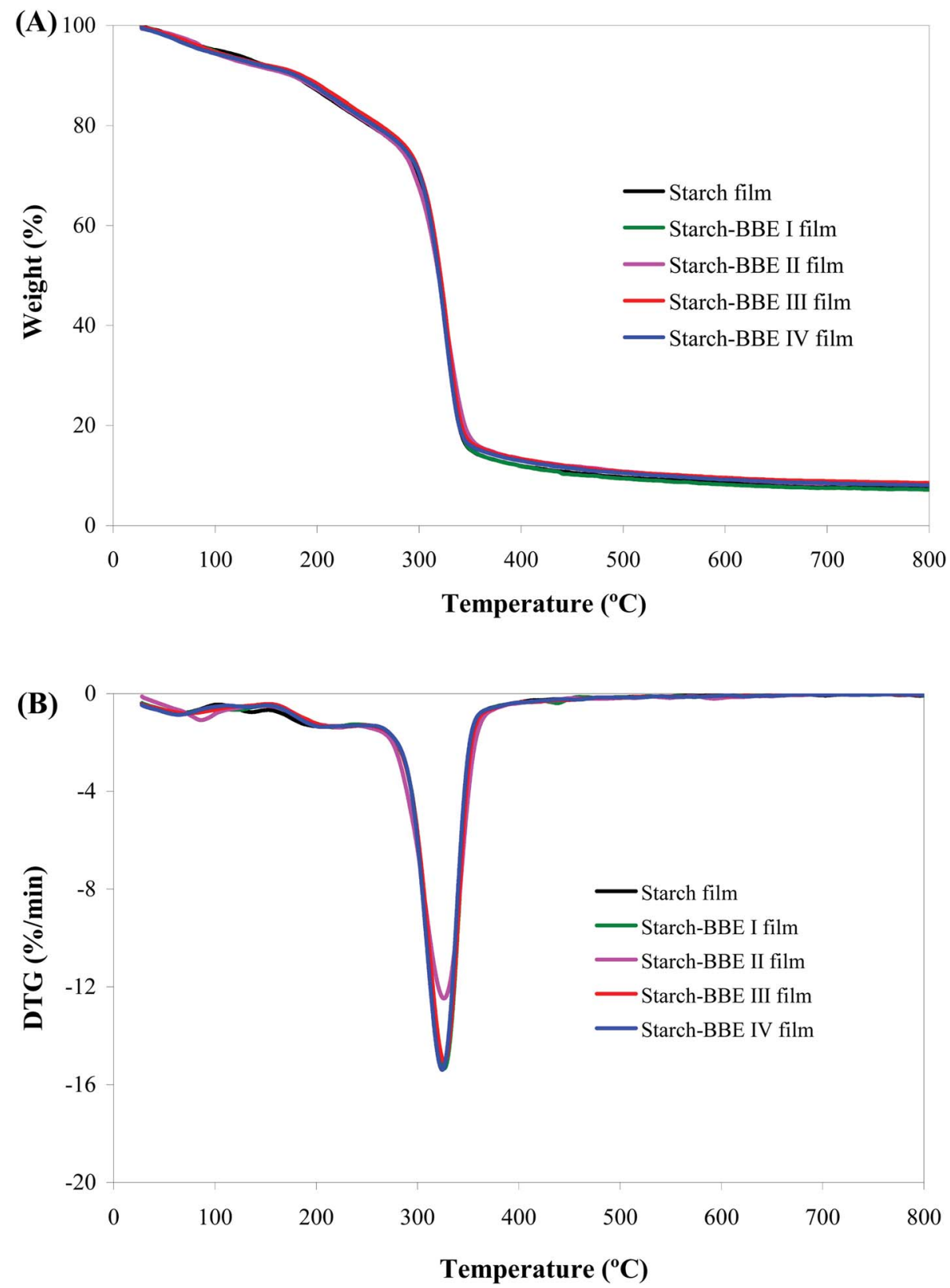

Fig. 6 TGA (A) and DTG (B) curves of starch and starch-BBE films.

unchanged by the addition of $L$. ruthenicum anthocyanins. Maciel et al. ${ }^{53}$ found the thermal degradation processes of chitosan-pectin films with and without anthocyanins were similar, indicating that the addition of anthocyanins did not affect the interactions between the polymers.

\subsection{Antioxidant ability of starch-BBE films}

The DPPH radical scavenging test was used to determinate the antioxidant ability of different films. As shown in Fig. 7, starch film had the lowest DPPH radical scavenging ability (only $7.97 \%$ at $5 \mathrm{mg} \mathrm{mL}^{-1}$ ). The weak antioxidant property of starch film was attributed to the abundant hydroxyl groups in the structure of starch and glycerol that could quench DPPH radical. ${ }^{20}$ The addition of BBE significantly increased the DPPH radical scavenging ability of the films $\left(24.39-75.01 \%\right.$ at $\left.5 \mathrm{mg} \mathrm{mL} \mathrm{mL}^{-1}\right)$. Notably, the DPPH radical scavenging ability of the films increased with the increase of BBE content. The enhanced DPPH radical scavenging ability of the films was mainly attributed to the presence of anthocyanins that possessed potent antioxidant property. Due to abundant phenolic hydroxyl groups in the structures, anthocyanins could donate free electron or hydrogen atoms to reactive free radicals, thereby quenching free radicals. ${ }^{54}$ Similarly, other researchers also found the antioxidant property of starch-based films were greatly enhanced by the addition of anthocyanin-rich L. ruthenicum extract and blackberry berry pulp. ${ }^{\mathbf{2 0 , 4 5}}$ 


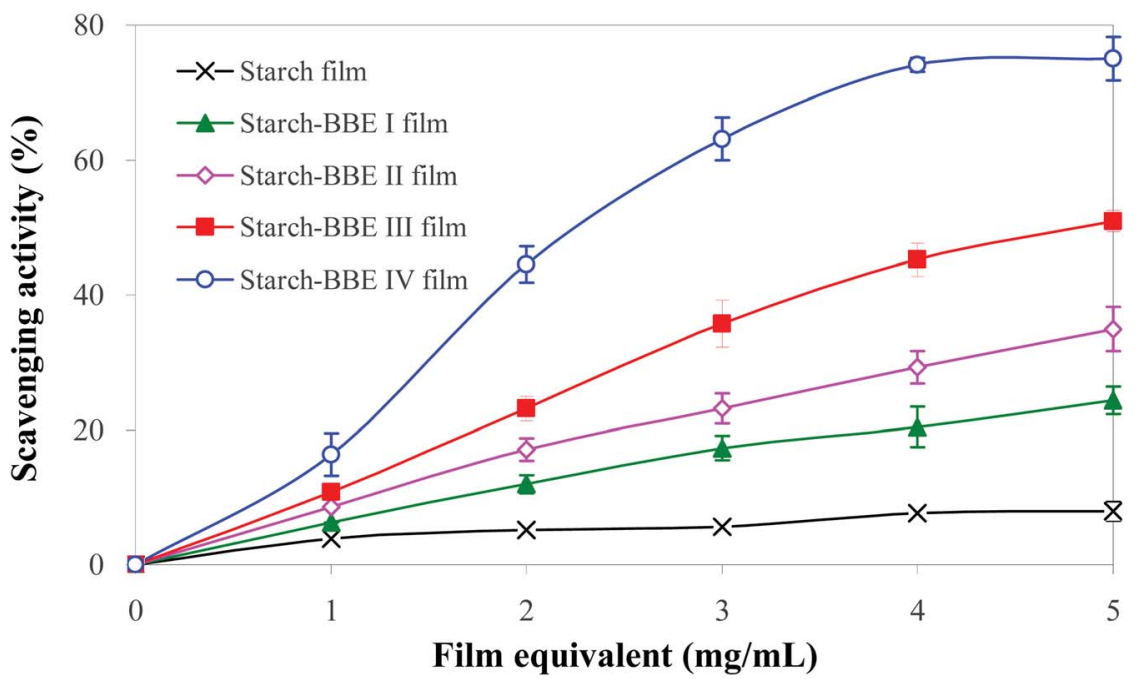

Fig. 7 DPPH radical scavenging activity of starch and starch-BBE films. Each value represents mean \pm SD of triplicates.

\subsection{3. pH-sensitive property of starch-BBE films}

In order to evaluate the $\mathrm{pH}$-sensitive property of the films, the representative starch-BBE I film was exposed to hydrogen chloride and ammonia gases, respectively (Fig. 8). The purple red color of the film gradually deepened in the hydrogen chloride gas, which was caused by the structure transformation of anthocyanins from quinonoidal anhydrobase (purple) into flavylium cation (red). Table $\mathrm{S} 1 \uparrow$ summarizes the color parameters of starch-BBE I film exposed to hydrogen chloride gas for different times. The gradually increased $a$ values confirmed the film became red. When exposed to ammonia gas, the color of the film quickly turned blue in $5 \mathrm{~min}$ and then changes into olive color after $35 \mathrm{~min}$, which was related with the structure transformation of anthocyanins from carbinol pseudobase to chalcone. ${ }^{38}$ Zhang et al. $^{39}$ suggested that ammonia gas firstly diffused into the film matrix and then hydrolyzed to produce hydroxyl ions, which made an alkaline environment for the film. Table $\mathrm{S} 2 \uparrow$ summarizes the color parameters of starch-BBE I film exposed to ammonia gas for different times. The film exhibited negative $b$ values before 35 min whereas presented positive $b$ values thereafter. In addition, the $b$ values of the film gradually increased with the extension of time. The changes in the color parameters were in good agreement with the observed color changes of the film. Above results indicated starch-BBE film was highly sensitive to $\mathrm{pH}$ changes. In other words, the film containing $1 \mathrm{wt} \%$ of BBE was sufficient to reflect the changes of environmental $\mathrm{pH}$. Wu et al. $^{34}$ found the chitosan/agarose film containing anthocyanins turned dark pink in less than 2 min in hydrogen chloride and turned purple after merely $2 \mathrm{~min}$ in ammonia gas, which was somewhat different from the results of our study. The differences in the color changes of the films could be due to the differences in the anthocyanin composition as well as the testing conditions (e.g. gas concentration and testing time). Our results suggested starch-BBE I film was $\mathrm{pH}$ sensitive and could be used as a $\mathrm{pH}$ indicator to monitor the freshness of food products.

\subsection{Application of starch-BBE films for monitoring the freshness of pork}

Fresh pork is highly susceptible to microbial contamination when exposed to the air, causing deterioration. Microbiological spoilage can produce a high level of TVB-N and change the $\mathrm{pH}$ value of pork. ${ }^{55}$ Since starch-BBE I film was $\mathrm{pH}$-sensitive, it was further used to monitor the freshness of pork. As shown in Table 4, the initial TVB-N value of fresh pork was $8.35 \mathrm{mg} / 100 \mathrm{~g}$. The TVB-N value of the pork increased to $10.35 \mathrm{mg} / 100 \mathrm{~g}$ when storage at $25{ }^{\circ} \mathrm{C}$ for $16 \mathrm{~h}$. Notably, the TVB-N value of the pork reached $17.16 \mathrm{mg} / 100 \mathrm{~g}$, which exceeded the limitation of Chinese Standard of GB 2707-2016 for edible pork (15 mg/100 $\mathrm{g})$. This suggested that the pork had seriously deteriorated at this stage. Similar results were also reported by other researchers, ${ }^{20,56}$ who also found pork rotted at $25{ }^{\circ} \mathrm{C}$ in $24 \mathrm{~h}$. With the extension of storage time to $48 \mathrm{~h}$, the TVB-N value of pork even reached $34.28 \mathrm{mg} / 100 \mathrm{~g}$. Meanwhile, starch-BBE I

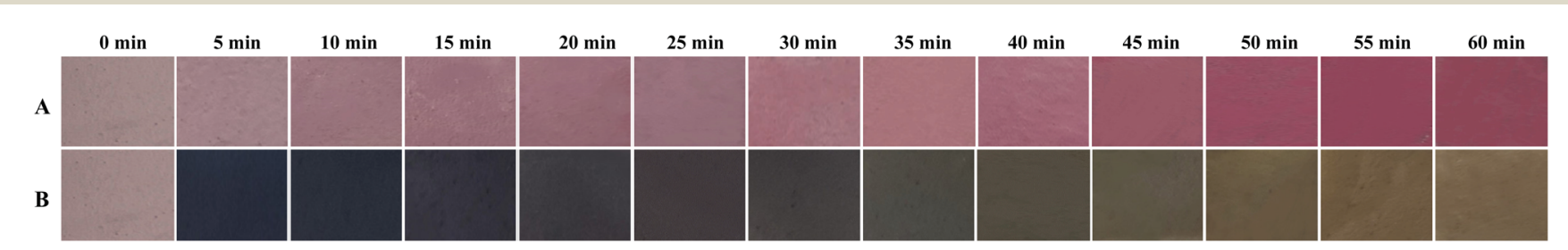

Fig. 8 Color changes of starch-BBE I film after being exposed to hydrogen chloride and ammonia gases for different times. 
Table 4 The change of TVB-N levels in the pork as well as the color response of starch-BBE I film at different storage times ${ }^{a}$

\begin{tabular}{lll}
\hline Time $(\mathrm{h})$ & TVB-N levels $(\mathrm{mg} / 100 \mathrm{~g})$ & Color response of starch-BBE I film \\
\hline
\end{tabular}

0

8

16

24

32

40

48

$$
8.35 \pm 0.61^{\mathrm{f}}
$$$$
8.77 \pm 0.78^{\mathrm{f}}
$$

$10.35 \pm 0.21^{\mathrm{e}}$

$17.16 \pm 1.15^{\mathrm{d}}$

$22.10 \pm 0.70^{\mathrm{c}}$

$28.01 \pm 1.59^{\mathrm{b}}$

$34.28 \pm 1.17^{\mathrm{a}}$

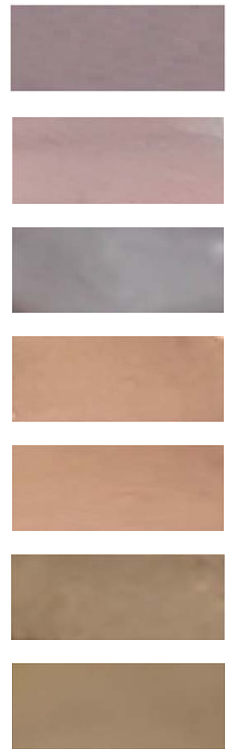

${ }^{a}$ Values are given as mean $\pm \operatorname{SD}(n=3)$. Different letters in the same column indicate significantly different $(p<0.05)$.

film showed significant color changes with the increase of the TVB-N value. Starch-BBE I film presented the purple/light color blue in the first $16 \mathrm{~h}$ and the orange color at $24-32 \mathrm{~h}$ and the yellow color thereafter. Table $\mathrm{S} 3 \dagger$ summarizes the color parameters of starch-BBE I film at different storage times. The film showed negative $b$ values before $24 \mathrm{~h}$ and positive $b$ value afterwards. In addition, the $b$ values of the film gradually increased with the extension of storage time. The changes in $b$ values of the film were consistent with its color changes. Therefore, starch-BBE I film could be used to effectively reflect the freshness of pork during storage.

\section{Conclusion}

Food packaging films with antioxidant and $\mathrm{pH}$-sensitive properties were successfully developed by incorporating different contents of BBE into cassava starch. Notably, $1 \mathrm{wt} \%$ of BBE addition improved the water vapor barrier property and TS of the film, which was because the incorporation of a low content of BBE created a more dense and compact film network. Due to the presence of anthocyanins in BBE, starch-BBE films showed enhanced UV-vis light barrier, antioxidant and $\mathrm{pH}$-sensitive properties. In future, starch-BBE films can be further used as intelligent films to monitor the freshness of different food products.

\section{Conflicts of interest}

There are no conflicts of interest to declare.

\section{Acknowledgements}

This work was supported by Grants-in-Aid for scientific research from the National Natural Science Foundation of China (No. 31571788), Innovation and Entrepreneurship Training Program for College Students in Jiangsu Province (No. 201811117061Y) and Northern Jiangsu Science and Technology Project (No. SZ-YC2018002).

\section{References}

1 A. Valdés, A. C. Mellinas, M. Ramos, N. Burgos, A. Jiménez and M. D. C. Garrigós, RSC Adv., 2015, 5, 40324-40335.

2 M. Kaya, S. Khadem, Y. S. Cakmak, M. Mujtaba, S. Ilk, L. Akyuz and E. Deligöz, RSC Adv., 2018, 8, 3941-3950.

3 R. Thakur, P. Pristijono, C. J. Scarlett, M. Bowyer, S. P. Singh and Q. V. Vuong, Int. J. Biol. Macromol., 2019, 132, 10791089.

4 E. Basiak, A. Lenart and F. Debeaufort, Int. J. Biol. Macromol., 2017, 98, 348-356.

5 S. Santacruz, C. Rivadeneira and M. Castro, Food Hydrocolloids, 2015, 49, 89-94.

6 C. L. Luchese, V. F. Abdalla, J. C. Spada and I. C. Tessaro, Food Hydrocolloids, 2018, 82, 209-218.

7 T. Niranjana Prabhu and K. Prashantha, Polym. Compos., 2018, 39, 2499-2522.

8 B. Iamareerat, M. Singh, M. B. Sadiq and A. K. Anal, J. Food Sci. Technol., 2018, 55, 1953-1959.

9 J. H. R. Llanos and C. C. Tadini, Int. J. Biol. Macromol., 2018, 107, 371-382. 
10 C. M. Jaramillo, T. J. Gutiérrez, S. Goyanes, C. Bernal and L. Famá, Carbohydr. Polym., 2016, 151, 150-159.

11 S. Silva, E. M. Costa, C. Calhau, R. M. Morais and M. E. Pintado, Crit. Rev. Food Sci. Nutr., 2017, 57, 3072-3083.

12 V. O. Silva, A. A. Freitas, A. L. Maçanita and F. H. Quina, J. Phys. Org. Chem., 2016, 29, 594-599.

13 H. E. Khoo, A. Azlan, S. T. Tang and S. M. Lim, Food Nutr. Res., 2017, 61, 1361779.

14 I. Choi, J. Y. Lee, M. Lacroix and J. Han, Food Chem., 2017, 218, 122-128.

15 V. A. Pereira Jr, I. N. Q. de Arruda and R. Stefani, Food Hydrocolloids, 2015, 43, 180-188.

16 R. Andretta, C. L. Luchese, I. C. Tessaro and J. C. Spada, Food Hydrocolloids, 2019, 93, 317-324.

17 L. B. Golasz, J. da Silva and S. B. da Silva, Food Sci. Technol., 2013, 33, 155-162.

18 C. L. Luchese, T. Garrido, J. C. Spada, I. C. Tessaro and K. de la Caba, Int. J. Biol. Macromol., 2018, 106, 834-839.

19 C. L. Luchese, J. Uranga, J. C. Spada, I. C. Tessaro and K. de la Caba, Int. J. Biol. Macromol., 2018, 115, 955-960.

20 Y. Qin, Y. Liu, H. Yong, J. Liu, X. Zhang and J. Liu, Int. J. Biol. Macromol., 2019, 134, 80-90.

21 P. Veiga-Santos, C. Ditchfield and C. C. Tadini, J. Appl. Polym. Sci., 2011, 120, 1069-1079.

22 S. Zhou, Z. Fang, Y. Lü, J. Chen, D. Liu and X. Ye, Food Chem., 2009, 112, 394-399.

23 C. Sun, H. Huang, C. Xu, X. Li and K. Chen, Plant Foods Hum. Nutr., 2013, 68, 97-106.

24 Z. Fang, Y. Zhang, Y. Lü, G. Ma, J. Chen, D. Liu and X. Ye, Food Chem., 2009, 113, 884-888.

25 J. Bao, Y. Cai, M. Sun, G. Wang and H. Corke, J. Agric. Food Chem., 2005, 53, 2327-2332.

26 Z. Fang, M. Zhang and L. Wang, Food Chem., 2007, 100, 845852.

27 B. Zhang, M. Kang, Q. Xie, B. Xu, C. Sun, K. Chen and Y. Wu, J. Agric. Food Chem., 2010, 59, 537-545.

28 W. Duan, S. Jin, G. Zhao and P. Sun, Food Sci. Technol., 2015, 35, 524-530.

29 J. Ge, P. Yue, J. Chi, J. Liang and X. Gao, Food Hydrocolloids, 2018, 74, 23-31.

30 X. Zhang, Y. Liu, H. Yong, Y. Qin, J. Liu and J. Liu, Food Hydrocolloids, 2019, 94, 80-92.

31 L. Stoll, T. M. H. Costa, A. Jablonski, S. H. Flôres and A. de Oliveira Rios, Food Bioprocess Technol., 2016, 9, 172-181.

32 X. Wang, H. Yong, L. Gao, L. Li, M. Jin and J. Liu, Food Hydrocolloids, 2019, 89, 56-66.

33 J. Liu, C. Meng, S. Liu, J. Kan and C. Jin, Food Hydrocolloids, 2017, 63, 457-466.
34 S. Wu, W. Wang, K. Yan, F. Ding, X. Shi, H. Deng and Y. Du, Carbohydr. Polym., 2018, 186, 236-242.

35 Z. Zhang, J. Li and L. Fan, Food Chem., 2019, 276, 451-457.

36 X. Zhang, H. Huang, Q. Zhang, F. Fan, C. Xu, C. Sun, X. Li and K. Chen, Int. J. Mol. Sci., 2015, 16, 12467-12481.

37 Q. Ma, T. Liang, L. Cao and L. Wang, Int. J. Biol. Macromol., 2018, 108, 576-584.

38 K. Halász and L. Csóka, Food Packaging and Shelf Life, 2018, 16, 185-193.

39 J. Zhang, X. Zou, X. Zhai, X. Huang, C. Jiang and M. Holmes, Food Chem., 2019, 272, 306-312.

40 H. Yong, X. Wang, R. Bai, Z. Miao, X. Zhang and J. Liu, Food Hydrocolloids, 2019, 90, 216-224.

41 J. Liu, X. Wang, F. Wen, S. Zhang, R. Shen, W. Jiang, J. Kan and C. Jin, Int. J. Biol. Macromol., 2016, 93, 107-116.

42 T. J. Gutiérrez, L. A. Toro-Márquez, D. Merino and J. R. Mendieta, Food Hydrocolloids, 2019, 89, 283-293.

43 X. Zhai, J. Shi, X. Zou, S. Wang, C. Jiang, J. Zhang, X. Huang, W. Zhang and M. Holmes, Food Hydrocolloids, 2017, 69, 308317.

44 M. Kurek, I. E. Garofulić, M. T. Bakić, M. Ščetar, V. D. Uzelac and K. Galić, Food Hydrocolloids, 2018, 84, 238-246.

45 G. F. Nogueira, C. T. Soares, R. Cavasini, F. M. Fakhouri and R. A. de Oliveira, Food Chem., 2019, 275, 417-425.

46 C. G. Vargas, T. M. H. Costa, A. de Oliveira Rios and S. H. Flôres, Food Hydrocolloids, 2017, 65, 96-106.

47 L. A. Toro-Márquez, D. Merino and T. J. Gutiérrez, Food Bioprocess Technol., 2018, 11, 1955-1973.

48 J. Peralta, C. M. Bitencourt-Cervi, V. B. Maciel, C. M. Yoshida and R. A. Carvalho, Food Packaging and Shelf Life, 2018, 19, 47-55.

49 A. Ashrafi, M. Jokar and A. M. Nafchi, Int. J. Biol. Macromol., 2018, 108, 444-454.

50 T. J. Gutiérrez and V. A. Alvarez, Food Hydrocolloids, 2018, 77, 407-420.

51 M. Koosha and S. Hamedi, Prog. Org. Coat., 2019, 127, 338347.

52 T. J. Gutiérrez, J. Suniaga, A. Monsalve and N. L. García, Food Hydrocolloids, 2016, 54, 234-244.

53 V. B. V. Maciel, C. M. P. Yoshida and T. T. Franco, Carbohydr. Polym., 2015, 132, 537-545.

54 A. Castaneda-Ovando, M. de Lourdes Pacheco-Hernández, M. E. Páez-Hernández, J. A. Rodríguez and C. A. GalánVidal, Food Chem., 2009, 113, 859-871.

55 J. Liu, H. Wang, P. Wang, M. Guo, S. Jiang, X. Li and S. Jiang, Food Hydrocolloids, 2018, 83, 34-142.

56 H. Yong, J. Liu, Y. Qin, R. Bai, X. Zhang and J. Liu, Int. J. Biol. Macromol., 2019, 137, 307-316. 